\title{
L'étude par diffraction de rayons $X$ des couches dures de carbonitrure de tungstène et de titanium produites par ablation laser réactive
}

\author{
N. Chitica, A. Lita, G. Marin, I.N. Mihailescu, M. Popescu*, C. Grivas** et A. Hatziapostolou** \\ Département de Lasers, Institut de Physique Atomique, BP. MG 6, 76900 Bucarest, Roumanie \\ * Institut de Physique et Technologie des Matériaux, BP. MG 7, 76900 Bucarest, Roumanie \\ ** FORTH-IESL, BP. 1527, Heraklion-71110, Crète, Grèce
}

Résumé: On a analysé, par diffraction de rayons $\mathrm{X}$, des couches minces de carbonitrures de tungstène et de titane obtenues par ablation laser réactive sur des cibles de tungstène et de titane dans une atmosphère de méthane (propane) à faible teneur en azote, sous basse pression. On a montré que les dépôts sont essentiellement constitués de carbures de tungstène et de nitrures de titane. On a obtenu des duretés d'environ $2600 \mathrm{GPa}$ pour les couches à base de tungstène et de $2200 \mathrm{GPa}$ pour les couches à base de titane.

\begin{abstract}
Thin films of tungsten and titanium carbonitrides have been prepared by reactive laser ablation of tungsten and titanium targets in a methane (propane) atmosphere with small amounts of nitrogen, at low pressure. The films have been investigated by X-ray diffraction. It was shown that the films are mainly composed of tungsten carbides and titanium nitrides. The microhardness of the samples reaches $2600 \mathrm{GPa}$ for tungsten based films and $2200 \mathrm{GPa}$ for titanium based films.
\end{abstract}

\section{INTRODUCTION}

Les couches de carbure et de nitrure de tungstène et de titane présentent un grand intérêt pour toutes les applications liées à leur capacité d'associer des propriétés physiques et chimiques tout à fait exceptionnelles: points de fusion très élevés, excellente résistance à l'usure, coefficients de frottement très faibles, conductivités thermiques et électriques élevées. Les couches sont utilisées pour les outils de coupe, comme barrières thermiques ou comme barrières de diffusion dans la technologie des semiconducteurs. Depuis quelques années on sait préparer des couches dures de bonne qualité à 1'aide des techniques laser [1,2]. Ce travail présente la préparation et l'étude des propriétés structurales des couches minces de carbonitrures de tungstène et de titane obtenues par ablation laser réactive.

\section{PREPARATION DES COUCHES}

L'ablation laser réactive est un procédé complexe qui fait intervenir l'extraction de matière d'une cible et la réaction chimique dans l'atmosphère qui entoure la cible et le collecteur. Sur le collecteur les produits de réaction forment une couche dont l'épaisseur augmente avec le nombre d'impulsions laser. Pour la préparation des couches on a utilisé des cibles en tungstène et en titane très purs $(99,6 \%)$ avec une atmosphère de méthane ou de propane $(99,97 \%)$ sous 
une pression comprise entre $0,5 \mu$ bar et $10 \mu$ bar. Avant l'introduction de ces hydrocarbure la chambre à réaction a été purgée à l'azote puis vidée jusqu'à $0,1 \mu$ bar.

Pour l'ablation on a utilisé un laser excimère $\mathrm{KrF}$ (Lambda Physik LPX200) fonctionnant à une fréquence de $10 \mathrm{~Hz}\left(\lambda=248 \mathrm{~nm}, \mathrm{t}_{\mathrm{FWHM}}<=30 \mathrm{~ns}\right)$. Pendant l'action du laser la cible a été mise en rotation afin d'éviter son percement ce qui minimise également l'expulsion du métal sous forme de gouttelettes. La fluence laser incidente était de $5 \mathrm{~J} . c m^{-2}$. Le collecteur en silicium était parallèle à la cible, à une distance comprise entre 50 et $70 \mathrm{~mm}$. L'épaisseur déposée, fonction de la distance cible-collecteur, était de 0,2 à $1 \mathrm{~A}$ /impulsion. Les couches ainsi obtenues ont une épaisseur estimée entre 50 et $100 \mathrm{~nm}$ et sont très dures et adhérentes au support. Le nombre des gouttelettes sur la surface est remarquablement petit.

\section{DIFFRACTION DES RAYONS $X$}

On a utilisé un diffractomètre Siemens Kristalloflex IV équipé d'un tube à anticathode de cuivre. Le faisceau diffracté a été filtré à l'aide d'un monochromateur à cristal courbe de graphite. Les rayons diffractées ont été enregistrés par un compteur à scintillation avec cristal de $\mathrm{NaI}(\mathrm{Tl})$ refroidi à l'eau. L'enregistrement des diagrammes de diffraction a été fait en balayant le domaine angulaire par pas de $0,02^{\circ}(2 \theta)$ avec un temps d'acquisition de $20 \mathrm{~s}$ par pas.

\section{RESULTATS}

\subsection{Couches de carbonitrures de tungstène}

La couche obtenue, de couleur grisâtre, correspond à la formation de carbure de tungtène. La figure 1 présente la région du diagramme de diffraction qui correspond aux raies les plus intenses du tungstène métallique et du carbure de tungstène.

On voit que la teneur en carbone des carbures augmente avec la pression des hydrocarbures ; les pics de diffraction sont remarquablement larges ce qui indique une grande finesse des cristallites. Leur dimension moyenne a été déterminée suivant le procédé de Scherrer. L'étude complémentaire par spectroscopie de photoélectrons (XPS) a révélé des traces d'oxides et la présence d'une certaine quantité d'azote dans les couches. C'est la raison pour laquelle nous considérons que nous avons formé des carbonitrures. La microdureté des couches, mesurée à l'aide d'un microdurimètre Vickers PMT-3, est d'autant plus grande que la teneur en carbone est élevée. Les valeurs les plus importantes de la dureté (2600 GPa) sont comparables à celles connues pour $\mathrm{W}_{2} \mathrm{C}(3000 \mathrm{GPa})$. Le Tableau 1 montre les résultats expérimentaux obtenus sur les couches à base de tungstène,

\begin{tabular}{|c|c|c|c|c|c|c|c|}
\hline $\begin{array}{c}\text { Repère } \\
\text { échantil. }\end{array}$ & $\mathbf{G a z}$ & $\begin{array}{c}\text { Pression } \\
\text { du gaz } \\
(\mu \mathrm{bar})\end{array}$ & $\begin{array}{c}\text { Distance } \\
\text { cible - } \\
\text { support } \\
(\mathbf{m m})\end{array}$ & $\begin{array}{c}\text { Nombre } \\
\text { d'impuls. }\end{array}$ & $\begin{array}{c}\mathbf{x} \\
\left(\mathbf{W C}_{\mathbf{x}}\right)\end{array}$ & $\begin{array}{c}\text { Dureté } \\
\mathbf{G P a}\end{array}$ & $\begin{array}{c}\mathbf{D}_{\text {(002) }} \\
(\mathbf{n m})\end{array}$ \\
\hline $\mathbf{A}$ & $\mathrm{CH}_{4}$ & $\mathbf{0 , 5}$ & $\mathbf{7 0}$ & $\mathbf{1 0 0 0 0}$ & $\mathbf{0 , 0 8}$ & $\mathbf{1 8 0 0}$ & $\mathbf{1 2 , 5}$ \\
$\mathrm{B}$ & $\mathrm{CH}_{\mathbf{4}}$ & $\mathbf{2}$ & $\mathbf{7 0}$ & $\mathbf{5 0 0 0}$ & $\mathbf{0 , 3 4}$ & $\mathbf{2 0 0 0}$ & $\mathbf{6 , 5}$ \\
$\mathrm{C}$ & $\mathrm{C}_{\mathbf{3}} \mathbf{H}_{\mathbf{8}}$ & 1 & $\mathbf{7 0}$ & 10000 & $\mathbf{0 , 3 4}$ & $\mathbf{2 6 0 0}$ & $\mathbf{7 , 5}$ \\
\hline
\end{tabular}

Tableau 1: Propriétés des couches de carbonitrure de tungstène preparées par ablation laser réactive. 


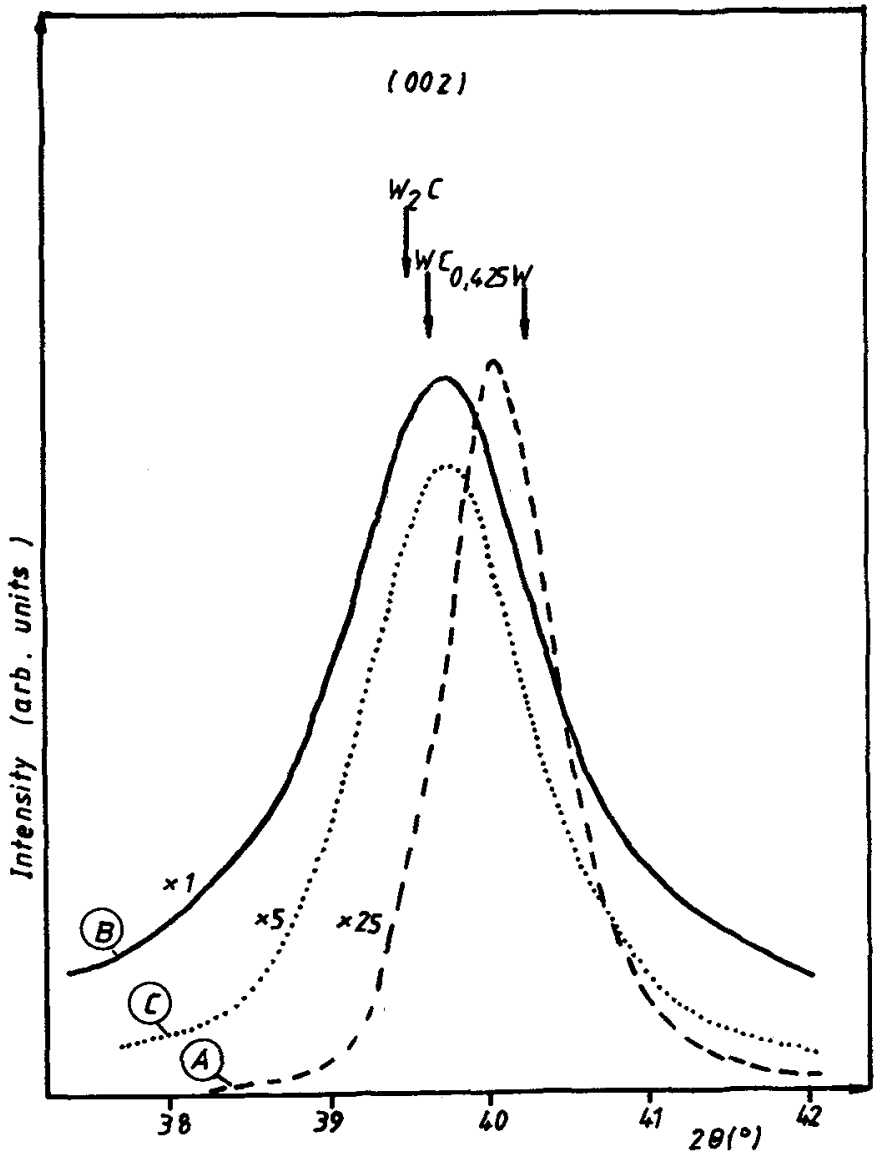

Figure 1: Diagramme de diffraction de rayons $\mathrm{X}$ montrant la présence de carbure de tungstène.

Figure 1: Tungsten carbide revealed in the films studied by X-ray diffraction

\subsection{Les couches de carbonitrures de titane}

La couleur jaune de ces couches indique la présence d'une quantité importante d'azote. Le spectre de diffraction montre la formation de la phase tetragonale de $\mathrm{Ti}_{2} \mathrm{~N}$ avec une orientation préférentielle des plans (200) des cristallites sur le support (figure 2).

Cette orientation préférentielle dans les couches de nitrures et de carbures a été observée par d'autres auteurs [3]. Le déplacement des raies (111) et (200) correspond au mélange de nitrures et carbures de titanium. La présence importante d'azote a éte observée par XPS. A partir de la position des raies on a estimé que la composition s'approchait de : $\operatorname{TiC}_{0,7} \mathrm{~N}_{0,3}$. Il est important de remarquer que l'on a constaté la présence de titane dans certaines couches pour lesquelles on a trouvé, par microscopie optique, des goutelettes en surface. 


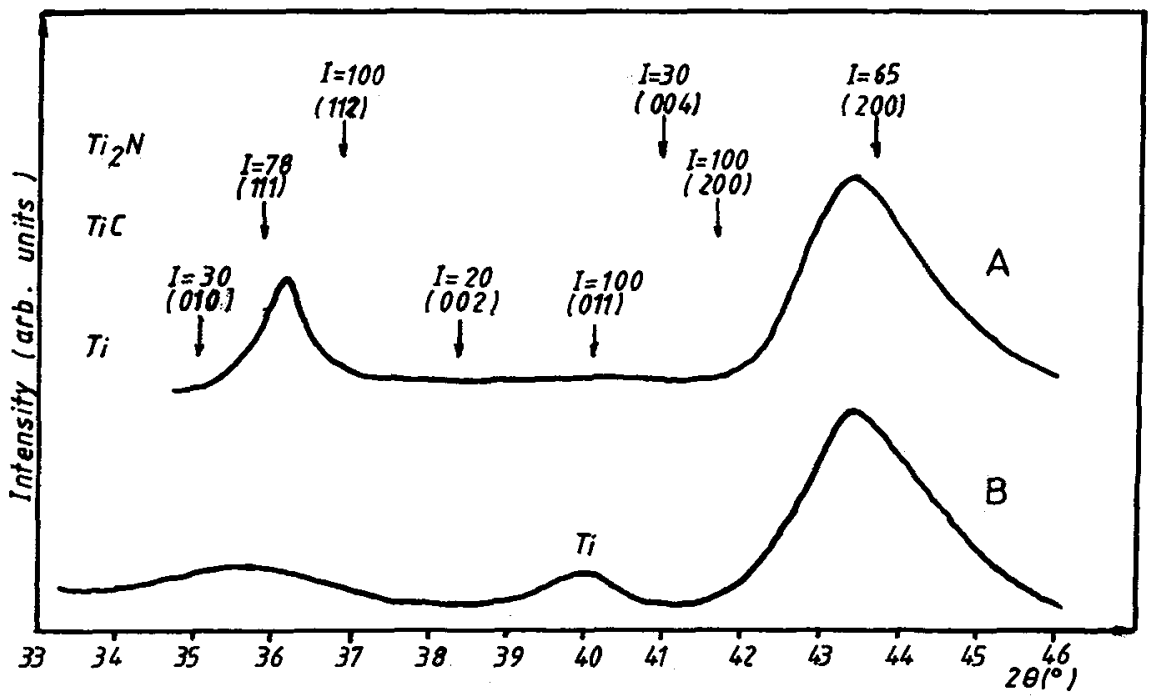

Figure 2: Diagramme de diffraction de rayons $\mathrm{X}$ pour deux échantillons de couches de carbonitrures de titanium déposées par ablation laser réactive.

Figure 2: X-ray diffraction patterns of two samples of titanium carbonitrides deposited by reactive laser ablation.

Les mesures de dureté Vickers donnent des valeurs de 1800 a $2000 \mathrm{GPa}$ qui sont très proches de celles indiquées dans la littérature: 1900-2200 GPa [3]. Le Tableau 2 présente les résultats des mesures effectuées sur ces couches à base de titane.

\begin{tabular}{|c|c|c|c|c|c|c|c|}
\hline $\begin{array}{c}\text { Repère } \\
\text { échantil.. }\end{array}$ & Gaz & $\begin{array}{c}\text { Pression } \\
\text { du gaz } \\
(\mu \mathrm{bar})\end{array}$ & $\begin{array}{c}\text { Distance } \\
\text { cible - } \\
\text { support } \\
(\mathrm{mm})\end{array}$ & $\begin{array}{c}\text { Nombre } \\
\text { d'impuls. }\end{array}$ & Compos. & $\begin{array}{c}\text { Dureté } \\
\text { (GPa) }\end{array}$ & Remarq- \\
\hline $\mathbf{A}$ & $\mathbf{C H}_{4}$ & $\mathbf{0 , 5}$ & $\mathbf{7 0}$ & $\mathbf{1 0 0 0 0}$ & $\mathbf{T i C}_{0,7} \mathbf{N}_{0,3}$ & $\mathbf{2 2 0 0}$ & $\begin{array}{c}\text { surf. } \\
\text { propre } \\
\text { surf. avec } \\
\text { goutelettes }\end{array}$ \\
\hline
\end{tabular}

Tableau 2: Propriétés structurales des couches de carbonitrures de titane

\section{DISCUSSION}

Nous pensons que les carbonitrures de tungstène et de titane se forment sur la surface du collecteur par la réaction entre les gaz adsorbés $\left(\mathrm{CH}_{4}, \mathrm{C}_{3} \mathrm{H}_{8}, \mathrm{~N}_{2}\right)$ et le flux du metal provenant de l'ablation. Un rôle important est joué par les agglomérations d'atomes (clusters) et par les gaz qui sont adsorbés à la surface du collecteur sous forme d'ions obtenus sous l'effet du plasma.

La présence d'une quantité importante d'azote pendant la carburation du titane, bien que la concentration de ce gaz soit faible, est due à la très forte réactivité du titane Par conséquent, le contrôle des traces d'azote dans l'atmosphère de réaction est essentiel pour la bonne reproductibilite de ces couches. Par contre dans le cas du carbure de tunstène l'action de l'azote dans des conditions identiques de réaction est à peu près négligeable. 
Les dimensions moyennes des cristallites de carbonitrures sont de l'ordre d'une dizaine de nanomètres ; cette taille est en relation inverse avec la pression du gaz dans la chambre de réaction.

\section{CONCLUSIONS}

Les couches minces déposées sur du silicium par ablation laser réactive en atmosphère d'hydrocarbure sont adhérentes et très dures. Selon la nature de la cible les couches peuvent contenir de l'azote provenant de l'azote résiduel de la chambre de réaction ; cette incorporation dépend du type de métal utilisé comme cible. Le titane montre une tendance forte à la nitruration. Les couches sont formées de carbonitrures à cristallites nanomètriques qui manifestent une certaine orientation préférentielle (200) sur le support.

\section{Remerciements}

Les auteurs N.C., A.L. et G.M. expriment leurs reconnaissance pour les possibilités offertes par U.E. dans le cadre du projet No. ERB 3563 PL 935005. Tous les auteurs remercient le Prof C. Fotakis de FORTH-IESL pour l'intérêt et le soutien permanent de ces recherches.

\section{References}

[1] Mihallescu I.N., Chitica N., Teodorescu V. S., De Giorgi M.L., Leggieri G., Luches A., Martino M., Perrone A. et Dubreuil B., J Vac. Sci. Technol. A11(5) (1993) 2577-2582.

[2] Mihailescu I.N., Chitica, Nistor L. C., Popescu M., Teodorescu V. S., Ursu I, Andrel A., Barborica A.; Luches A., De Giorgi M.L., Perrone A., Dubreuil B. et Hermann J., J Appl. Phys. 74(9) (1993) 5781-5789.

[3] Bertoncello R., Casagrande A., Casarin M., Glisenti A., Lanzoni E., Mirenghi L, et

Tondello E., Surf. Interf. Anal. 18 (1992) 525-531. 